\title{
Performance of cement-based sensors with CNT for strain sensing
}

\section{Carmen Camacho-Ballesta}

Civil Engineer, PhD Student, Department of Civil Engineering, Universidad de Alicante, San Vicente del Raspeig, Spain

Emilio Zornoza

Associate Professor, Department of Civil Engineering, Universidad de Alicante, San Vicente del Raspeig, Spain

\section{Pedro Garcés}

Professor, Department of Civil Engineering, Universidad de Alicante, San Vicente del Raspeig, Spain

Strain-sensing functions of Portland cement pastes with different dosages of carbon nanotubes (CNT) are systematically studied. This is one of the relevant functions that multifunctional composites are able to develop when a conductive addition is included in the cement matrix. Strain sensing refers to the ability of a structural material to sense its own condition, such as strain or stress, through the piezoresistive behaviour (change of volume electrical resistivity when it is subjected to a force). CNTs, which possess exceptional electromechanical properties, are considered as a promising addition for practical applications such as structural health and load monitoring. Nevertheless, there is still an important challenge regarding CNT cement composite fabrication: achieving their effective dispersion. This point is addressed by means of a comparison between different physical and chemical dispersion methods. The technique used for that purpose is light-scattering particle size analysis. After selecting the optimal dispersing procedure, the effect on the strain-sensing properties of CNT reinforced cement pastes was studied for the following variables: CNT dosage, curing age, current intensity, loading rate and maximum stress applied. All these parameters are discussed taking gauge factor value as the reference.

\section{Notation}

D material's density

E elastic modulus

$l_{0} \quad$ initial length

$M_{\mathrm{d}} \quad$ dry mass after drying in oven at $105 \pm 5^{\circ} \mathrm{C}$

$M_{\mathrm{s}} \quad$ saturated mass

$M_{\mathrm{s}}^{\mathrm{hb}} \quad$ saturated mass measured in hydrostatic balance

$P \quad$ material's total porosity

$R_{0} \quad$ initial electrical resistance

$\Delta l \quad$ specimen's deformation

$\Delta R \quad$ change on electrical resistance

$\varepsilon \quad$ unit strain

$\sigma \quad$ stress

\section{Introduction}

In the last few years, advances in nanotechnology have been conducted to obtain new nanoscale fibres with outstanding electromechanical properties, specifically carbon nanotubes (CNT). Thanks to their very low electrical resistivity $\left(5 \times 10^{-8}-2 \times 10^{-6} \Omega \mathrm{m}\right.$, which is similar or superior to that of copper (Kim et al., 2014)) they can be considered a conductive admixture for cement, making the development of new multifunctional, high-performance, advanced sensing, cement-based nanocomposites possible.
The strain-sensing function refers to the ability of a structural material to sense its own condition, such as strain or stress (which simply relates to the strain in the elastic regime) (Chung, 2012). When subjected to stress/strain, their electrical properties change, expressing a linear and reversible response, called piezoresistive response. Previous work has indicated that the contact resistivity between the matrix and the conductive admixture is responsible for the piezoresistive phenomena (Wen and Chung, 2006). In a practical way, if a longitudinal compressive stress is applied, the electrical resistance in that direction will be reduced. However, if the material is in tension, a contrary effect will be produced, that is, an increase in the electrical resistance will be registered. As both effects are reversible in the material's elastic range, the electrical resistance comes back to its initial value once the load is removed. This sensing ability can be useful for structural vibration control, load monitoring and structural health monitoring (Galao et al., 2014).

Few studies have been performed on the strain-sensing ability of cement-based composites with the addition of CNTs. Li et al. (2007) conducted experiments using acid-treated CNTs and untreated CNTs as reinforcement and concluded that both types can decrease the electrical resistivity and improve pressuresensitive properties of cement composites. Azhari and Banthia 
(2012) studied two types of cement-based sensors, one with just carbon fibres and the other carrying a hybrid of both carbon fibres and nanotubes. They found that both types showed piezoresistive response, providing the hybrid sensors with a better quality signal and increased sensitivity over sensors carrying carbon fibre alone. Yu and Kwon (2009) also found a piezoresistive behaviour for cement pastes reinforced with multiwalled carbon nanotubes (MWCNTs). Han et al. (2009) investigated a self-sensing CNT/cement composite for traffic monitoring in laboratory tests and road tests. Results showed that under repeated compressive loading the electrical resistivity decreases upon loading and increases when unloaded, leading to the conclusion that this nanocomposite can detect vehicular loads through remarkable changes in electrical resistance. Later, Han et al. investigated the effect of the MWCNT content and water/ cement ratio on the piezoresistive sensitivity of composites. They examined cement nanocomposites with amounts of MWCNT of $0 \cdot 05,0 \cdot 1$ and $1 \mathrm{wt} \%$ (Han et al., 2012). Experimental results concluded that the composite with $0 \cdot 1 \mathrm{wt} \%$ of MWCNT presents the best sensing property. Luo et al. (2011) used cured MWCNT reinforced cement-based composites with $0 \cdot 1 \mathrm{wt} \%$ and $0.5 \mathrm{wt} \%$ MWCNT and registered good piezoresistivity results and strain sensitivity for both samples, although the trendline of fractional change in resistivity presented better stability for amounts of $0.5 \mathrm{wt} \%$. More recently, Konsta-Gdoutos and Aza (2014) investigated the resistivity of cementitious composites reinforced with CNT and carbon nanofibres (CNF). They found the addition of CNTs and CNFs at an amount of $0 \cdot 1 \mathrm{wt} \%$ and $0.3 \mathrm{wt} \%$ with respect to cement mass induced a decrease in electrical resistance, with the nanocomposites containing $0 \cdot 1 \mathrm{wt} \%$ CNTs yielding better electrical properties. Furthermore, their strain-sensing results confirmed that nanocomposites, reinforced with $0 \cdot 1 \mathrm{wt} \%$ CNTs and CNFs, exhibited an increased change in resistivity, which is indicative of the amplified sensitivity of the material in strain sensing.

Usually the strain-sensing sensitivity is measured using the gauge factor (GF), which can be defined as the fractional change in electrical resistance per strain unit. This parameter can be calculated as follows

1. $\mathrm{GF}=\frac{\Delta R / R_{0}}{\Delta l / l_{0}}=\frac{\Delta R / R_{0}}{\varepsilon}$

where $\Delta R$ is the change in electrical resistance, $R_{0}$ is the initial electrical resistance, $\Delta l$ is the specimen's deformation, $l_{0}$ is the initial length and $\varepsilon$ is the unit strain.

The main problem of CNTs is their difficult dispersion because of the highly attractive van der Waals forces between the CNT particles that tend to cause agglomeration (Figure 1). The insufficient dispersion of CNTs has been cited as a key diminishing factor for the performance of CNT-cement composites, as poor dispersion of CNTs leads to the formation of many defects in the nanocomposite and limits the mechanical

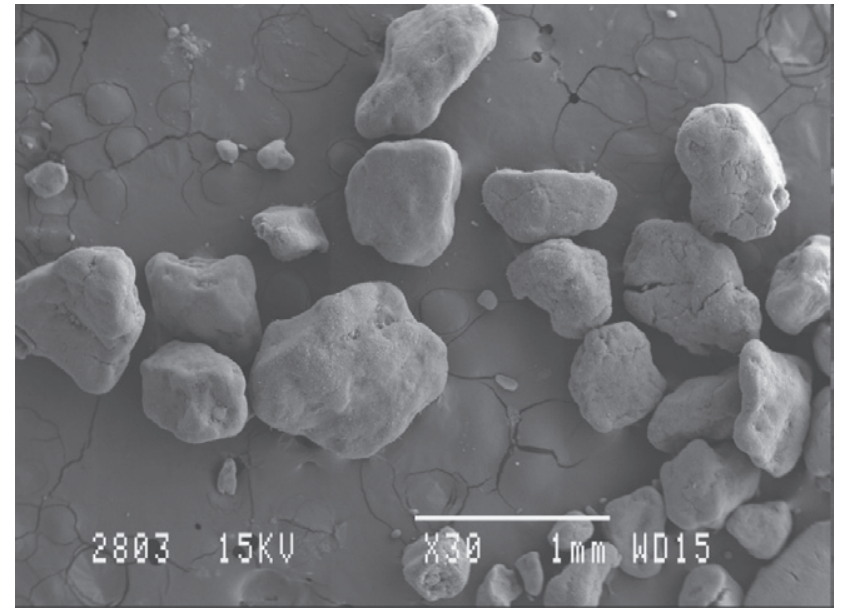

(a)

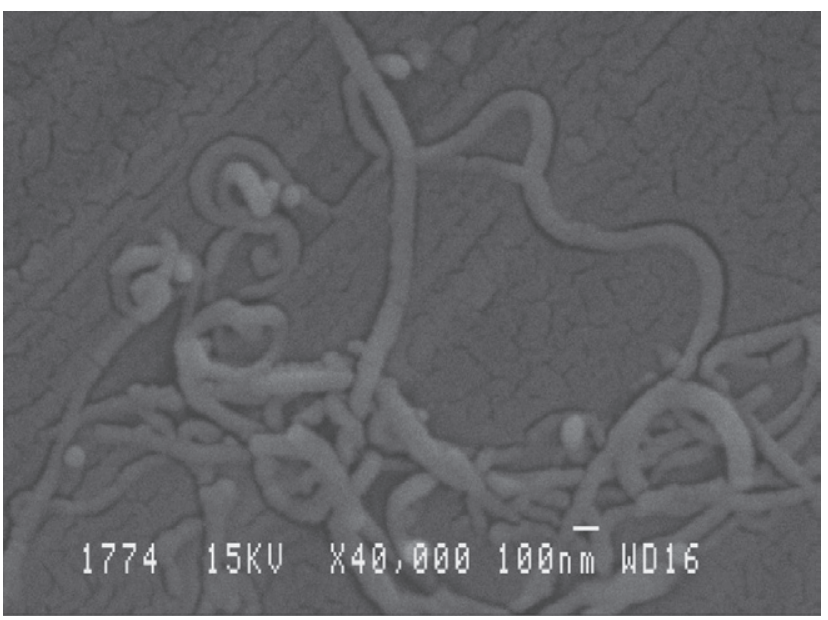

(b)

Figure 1. Scanning electron microscope EM images of (a) non-dispersed, as received, CNT and (b) dispersed CNT

properties of the composites. On the contrary, better dispersion of the CNTs may result in a higher interfacial contact area between the CNTs and the matrix, as well as more evenly distributed stresses in the composites (Chen et al., 2011). According to the literature, there are many different dispersion technologies, but all of them can be classified into chemical or physical techniques (Parveen et al., 2013). The basic physical technique used for CNT is ultrasonication, which is often used in combination with other chemical processes. Chemical approaches are designed as either a covalent treatment (CNT functionalisation), as researched previously for other carbon materials (Catala et al., 2011), or a non-covalent treatment (dispersing chemical groups are physically attached onto the CNT surface). However, it has been reported that functionalisation might introduce structural defects resulting in inferior properties of the treated CNTs (Coleman et al., 2006). Luo et al. (2009) assessed the dispersion capability in aqueous solution of five surfactants: sodium dodecyl benzene sulfonate, 
Triton X-100, sodium deoxycholate, Arabic gum and cetyltrimethyl ammonium bromide. The best results were obtained from a combination of the first two with a mixing ratio of $3: 1$, respectively. Cement composites reinforced with MWCNT subjected to that treatment had balanced mechanical and electrical properties. Collins et al. (2012) also investigated the dispersion of CNTs with different dispersants: air entrainer, styrene butadiene rubber, polycarboxylates, calcium naphthalene sulfonate and lignosulfonate formulations, achieving the best results for polycarboxylate additives.

Therefore, it is essential to integrate dispersion techniques into the fabrication of the CNT-cement composites for more effective use of CNTs as reinforcements. This point has been addressed first in the present research. Then a systematic study of strain-sensing properties in CNT reinforced cement pastes was performed. The effect of several variables on this function was studied, for example, CNT dosage, cement pastes curing age, current intensity, loading rate or maximum stress applied.

\section{Experimental programme and materials}

\section{Materials and sample fabrication}

Cement pastes were used for mechanical and physical tests (compressive and bending strengths, porosity and density) and strain-sensing tests, while fluid suspensions in water were prepared for dispersion trials. The materials used in this research were: Portland cement type EN 197-1 CEM I 52.5 R; multiwall carbon nanotubes (MWCNT, Baytubes ${ }^{\circledR}$ C 70P), supplied by Bayer MaterialScience, S.A., whose characteristic properties are outlined in Table 1; distilled water; and Sikament-200 R, a commercial superplasticiser, supplied by Sika España. The following types of surfactants/dispersants were chosen because of their demonstrated solubilisation capability on MWCNTs (Collins et al., 2012; Luo et al., 2009)

sodium dodecyl benzene sulfonate (SDBS)

Triton X-100 (TX-100)

\begin{tabular}{lcc}
\hline Properties & Value & Unit \\
\hline C-Purity & $\geq 95$ & $\mathrm{wt} \%$ \\
Free amorphous carbon & - & \\
Outer mean diameter & $\sim 13$ & $\mathrm{~nm}$ \\
Inner mean diameter & $\sim 4$ & $\mathrm{~nm}$ \\
Length & $>1$ & $\mu \mathrm{m}$ \\
Bulk density & $45-95$ & $\mathrm{~kg} / \mathrm{m}^{3}$ \\
Elastic modulus & 3596 & $\mathrm{MPa}$ \\
Tension at break & 72.9 & $\mathrm{MPa}$ \\
Elongation at break & 10.7 & $\%$ \\
Izod-impact at $23^{\circ} \mathrm{C}$ & 103 & $\mathrm{~J} / \mathrm{m}$
\end{tabular}

Table 1. Properties of Baytubes $®$ C 70P multiwall CNT
- polycarboxylate 1 (PC1), specifically Sika Viscocrete-20 HE, supplied by Sika España

- polycarboxylate 2 (PC2), specifically Glenium ACE 425, supplied by Basf Construction Chemicals España.

The water/cement ratio (w/c) for all pastes was 0.5 and CNT dosages were $0,0 \cdot 05,0 \cdot 10,0 \cdot 25$ and $0 \cdot 50 \%$ by cement mass. The dosages of superplasticiser were used for pastes according to previous research (Camacho-Ballesta et al., 2014), for obtaining the same workability for all pastes. Thus the specific quantity of plasticiser was $0,0 \cdot 4,0.5,0.9$ and $2.2 \%$ by cement mass, for CNT dosages of $0,0 \cdot 05,0 \cdot 10,0 \cdot 25$ and $0.50 \%$, respectively.

Carbon nanotube dispersions for pastes were done according to a previously checked method in polymer composites with CNF addition (Bortz et al., 2011) and followed these steps: $\mathrm{CNT}$ and distilled water were poured into a high-shear mixer for $10 \mathrm{~min}$ and afterwards an ultrasound treatment was applied for 5 min using an ultrasound device model Hielschier UP200S. The resulting dispersion was mixed with cement and plasticiser in a laboratory mixer for $5 \mathrm{~min}$. Pastes were fabricated in laboratory conditions: $20^{\circ} \mathrm{C}$ temperature and $65 \%$ relative humidity $(\mathrm{RH})$. This dispersion method has been used successfully in CNF cement composites (Baeza et al., 2013b; Galao et al., 2012).

For each dosage three prismatic specimens of $40 \times 40 \times$ $160 \mathrm{~mm}^{3}$ were fabricated according to AENOR Standard UNE EN 196-3:2005 + A1:2009. The first specimen was used for mechanical and other characterisation tests (cured in water for $28 \mathrm{~d}$ ), the two other specimens for piezoresistive sensing tests (cured in water until the first test date, that is $14 \mathrm{~d}$, and afterwards in $100 \% \mathrm{RH}$ condition).

\section{Dispersion trials}

A battery of suspensions was prepared by combination of different known methods for dispersing CNTs. They can be summarised as follows

no treatment

10 min mixer

10 min mixer +5 min ultrasound

$0 \cdot 25 \%$ of polycarboxylate 1

- $0.25 \%$ of polycarboxylate 2

- $0 \cdot 25 \%$ of sodium dodecyl benzene sulfonate

$0 \cdot 25 \%$ of Triton X-100

- 5 min ultrasound $+0,0 \cdot 25,0.50$ and $1 \%$ of polycarboxylate $1+$ ultrasound bath

- 5 min ultrasound $+0,0 \cdot 25,0 \cdot 50$ and $1 \%$ of polycarboxylate $2+$ ultrasound bath

- 5 min ultrasound $+0,0 \cdot 25,0 \cdot 50$ and $1 \%$ of sodium dodecyl benzene sulfonate + ultrasound bath

- 5 min ultrasound $+0,0 \cdot 25,0 \cdot 50$ and $1 \%$ of Triton $\mathrm{X}-100+$ ultrasound bath. 
For this study, as in previous dispersion studies, the dosage of CNT was established on $0.05 \%$ of cement mass (Collins et al., 2012). The suspensions were prepared by manual agitation of CNTs in $500 \mathrm{ml}$ of water with a glass bar followed by indicated treatments for each case. An additional ultrasound bath was also applied to the last four dispersions to guarantee the complete dispersant dissolution. Then a sample of about $10 \mathrm{ml}$ was taken to be tested, keeping the suspension free of movements on a stable surface to observe the decantation process.

The instrument selected to evaluate the dispersions was a Coulter LS230, a light-scattering particle size analyser that measures particles from $0.04 \mu \mathrm{m}$ to $2000 \mu \mathrm{m}$. It uses the diffraction of laser light by CNTs as the main source of information about particle size.

\section{Mechanical properties and other characterisation tests}

At 28-d age mechanical tests were conducted in laboratory conditions following the analogous procedure to AENOR Standard UNE EN 196-1:2005 for all pastes, with a ME-402/20 press machine (Servosis, S.A., Spain) to determine bending and compression strength for each dosage. Previously the non-destructive ultrasonic pulse velocity (UPV) data had been registered.

Porosity $(P)$ and density $(D)$ were calculated from at least two samples collected from the preceding mechanical tests. Apparent density was calculated as follows

$$
D=M_{\mathrm{d}} /\left(M_{\mathrm{s}}-M_{\mathrm{s}}^{\mathrm{hb}}\right)
$$

where $M_{\mathrm{d}}$ is dry mass after drying in an oven at $105 \pm 5^{\circ} \mathrm{C}, M_{\mathrm{s}}$ is saturated mass and $M_{\mathrm{s}}^{\mathrm{hb}}$ is saturated mass measured in hydrostatic balance.

Porosity was obtained as follows

$$
P(\%)=\left(M_{\mathrm{s}}-M_{\mathrm{d}}\right) /\left(M_{\mathrm{s}}-M_{\mathrm{s}}^{\mathrm{hb}}\right) \times 100
$$

Porosity was also characterised by mercury intrusion porosimetry, using an Autopore IV 9500 V1.05 (Micromeritics Instrument Corporation).

\section{Experimental set-up for measuring strain sensing}

After the curing period samples were externally dried and silver electrically conductive paint (Pelco Conductive Silver 187) was applied around the perimeter at four interior planes which were parallel to the end surfaces. Additionally, four copper wires were wrapped around each silver painted perimeter, in order to form four electrical contacts, as needed for the four-probe method (Figure 2).

Each test consisted of at least four consecutive loadingunloading cycles (compressive). According to Equation 1, the gauge factor (GF) was calculated for each semi-cycle (i.e. only

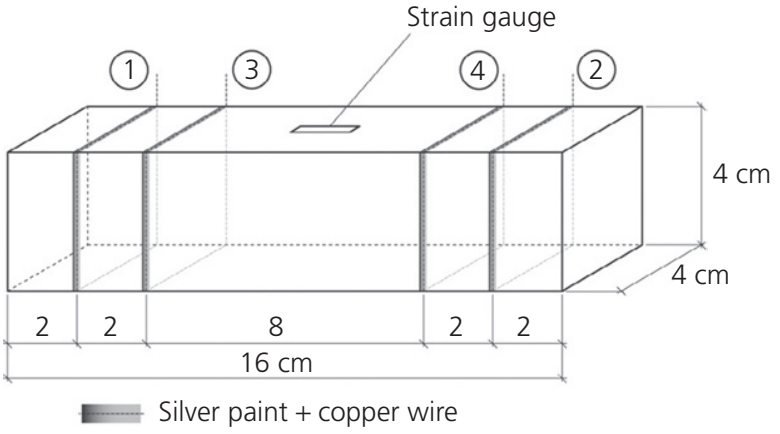

Figure 2. Electrical contacts configuration: 1 and 2 are contacts for current input and 3 and 4 are contacts for voltage measurement

the loading or unloading part of each complete cycle) and for each completed cycle (considering both loading and unloading parts). The following parameters were systematically studied.

- CNT dosage: pastes with $0 \%, 0 \cdot 05 \%, 0 \cdot 10 \%, 0 \cdot 25 \%$ and $0 \cdot 50 \%$ CNT by cement mass.

- Curing age: samples were tested at 14 and $28 \mathrm{~d}$. Between each test the samples were kept in a desiccator at room temperature and water saturated ambient $(100 \% \mathrm{RH})$.

- Current intensity: $0 \cdot 1,1$ and $10 \mathrm{~mA}$.

- Maximum load of each cycle: 3, 6 and $9 \mathrm{kN}$ loads, corresponding to stress values of $1 \cdot 9,3 \cdot 8$ and $5 \cdot 6 \mathrm{MPa}$, respectively.

- Loading rates (velocity): $0 \cdot 05,0 \cdot 10$ and $0 \cdot 20 \mathrm{kN} / \mathrm{s}$ for a $3 \mathrm{kN}$ maximum load, and $0 \cdot 10,0 \cdot 20$ and $0 \cdot 40 \mathrm{kN} / \mathrm{s}$ for 6 and $9 \mathrm{kN}$ maximum loads.

The strain-sensing tests were carried out according to the diagram in Figure 3. For the electrical resistance measures during the tests, an electrical current intensity was set between the outer contacts by means of a DC current source (model Keithley 6220). The voltage was measured between the inner contacts using a digital multimeter (Keithley Model, 2002). Hence resistance may be calculated by applying Ohm's law. Tests were conducted on an electromechanical press model Microtest $10 t / 2 t$ (Servosis, S.A., Spain). Loads were applied on the smallest faces, that is in the longitudinal direction, and the specimens were perfectly centred on the press to obtain a uniaxial and uniform stress distribution. Strain was permanently monitored with a Vishay P3 extensometer and strain gauges located in the middle point of the lateral sides of the samples, and oriented in the longitudinal direction (the same as the loading direction).

Before and after every test performed on each sample, their masses were controlled. As the mass losses were always below $1 \%$ of the initial mass, the water saturation state can be considered constant for all experimental phases; that is, electrical resistance changes are not expected to occur due to water loss (ionic conductivity). Additionally, before any load was applied, 


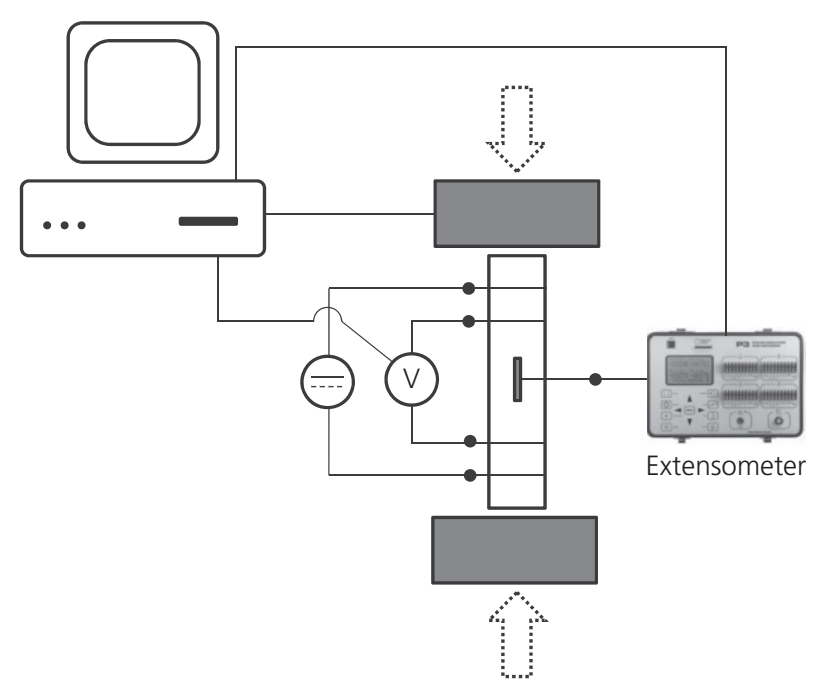

Figure 3. Diagram of strain-sensing test set-up. Current source and multimeter make it possible to obtain electric resistance values, while extensometer and press provide mechanical data for unit strain and applied stress, respectively

the initial electrical resistance was measured. Therefore the initial resistance was controlled in order to calculate the GF after loading the samples.

\section{Results and discussion}

Mechanical properties and characterisation tests on CNT cement pastes

Previous compressive strength tests were conducted to determine the loading limits, with the first specimen of each CNT dosage. In order to guarantee an elastic behaviour, the loading conditions during later strain-sensing tests should be below 30\% of compressive strength (according to UNE-EN 12390-13:2014). Table 2 shows the specimens' compressive strength mean values. It also includes results of bending strength, ultrasonic pulse velocity, porosity and apparent density for all CNT dosages. Slight

\begin{tabular}{lcrrrr}
\hline $\begin{array}{l}\text { CNT } \\
\text { dosage: \% } \\
\text { by cement } \\
\text { mass }\end{array}$ & $\begin{array}{c}\text { Compressive } \\
\text { strength: } \\
\mathrm{N} / \mathrm{mm}^{2}\end{array}$ & $\begin{array}{r}\text { Bending } \\
\text { strength: } \\
\mathrm{N} / \mathrm{mm}^{2}\end{array}$ & $\begin{array}{c}\text { UPV: } \\
\mathrm{km} / \mathrm{s}\end{array}$ & $\begin{array}{c}\text { Porosity: } \\
\%\end{array}$ & $\begin{array}{r}\text { Density: } \\
\mathrm{g} / \mathrm{cm}^{3}\end{array}$ \\
\hline 0.00 & $49 \cdot 2$ & $3 \cdot 1$ & 3.46 & $44 \cdot 2$ & 1.43 \\
0.05 & 51.2 & 3.2 & 3.55 & 43.5 & 1.50 \\
0.10 & 52.4 & 4.4 & 3.51 & 44.2 & 1.45 \\
0.25 & 53.0 & 3.4 & 3.52 & 44.7 & 1.45 \\
0.50 & 49.0 & 3.7 & 3.40 & 44.7 & 1.44
\end{tabular}

Table 2. Compressive strength, bending strength, ultrasonic pulse velocity (UPV), porosity and density of different CNT dosage pastes performed at $28 \mathrm{~d}$ curing time variations have been observed for different CNT additions. This trend is consistent with previous research (Camacho-Ballesta et al., 2014). Figure 4 reflects the results obtained from mercury intrusion porosimetry; no meaningful differences are shown for the different dosages analysed.

\section{Evaluation of dispersions}

When analysing dispersion effectiveness, it was expected that the higher the applied treatment efficacy, the smaller the particle size obtained from the processed light-scattering signal would be. This would mean that CNTs agglomerations are divided into smaller ensembles of CNTs particles, achieving complete dispersion when CNTs can be individually detected. This hypothesis allows the differences found among all applied treatments to be discussed qualitatively. Figure 5 and Figure 6 show the results provided by a light-scattering analyser for applied physical and chemical treatments, respectively. At first sight it seems that physical treatments have a greater dispersant ability than chemical treatments. According to Figure 5, all different mechanical treatments show a particle size smaller than no treatment. Mixer treatment has an indisputable dispersant effect on CNTs by itself, but has no significant contribution when it is combined with subsequent ultrasound treatment, probably owing to a higher energy quantity transferred to CNT conglomerates by ultrasonication technique compared with the mixer treatment. Regarding chemical treatments alone (Figure 6), surfactants would present a certain higher dispersant ability than two polycarboxylates, showing the SDBS the enhanced effect. Nevertheless if a combined dispersant/ultrasonic treatment is

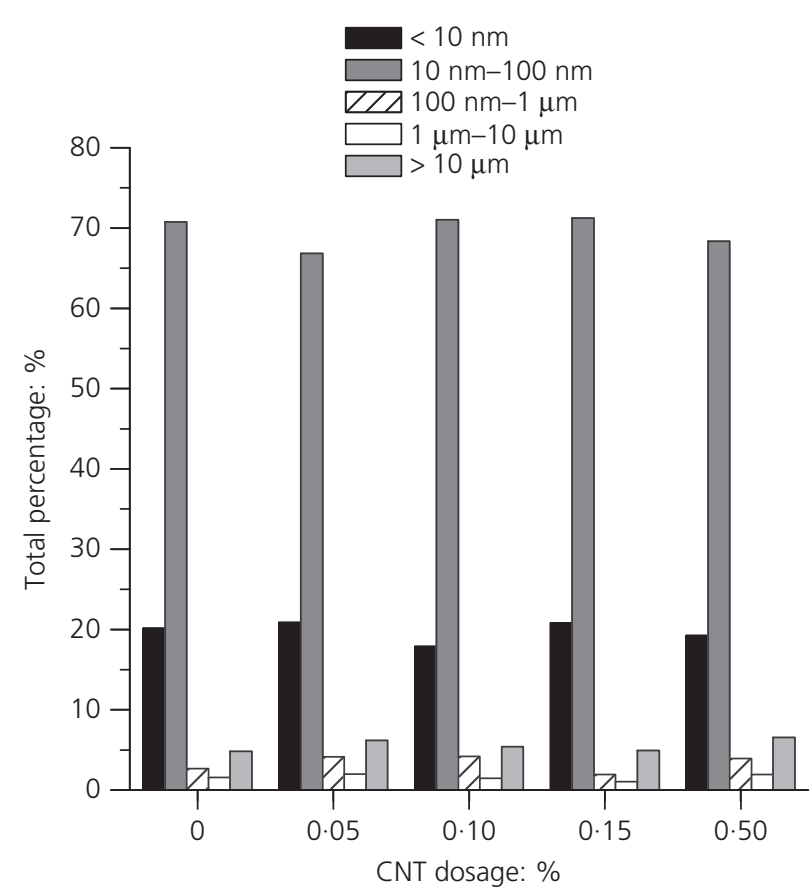

Figure 4. Pore diameter distribution for CNT cement pastes resulting from mercury intrusion porosimetry tests 


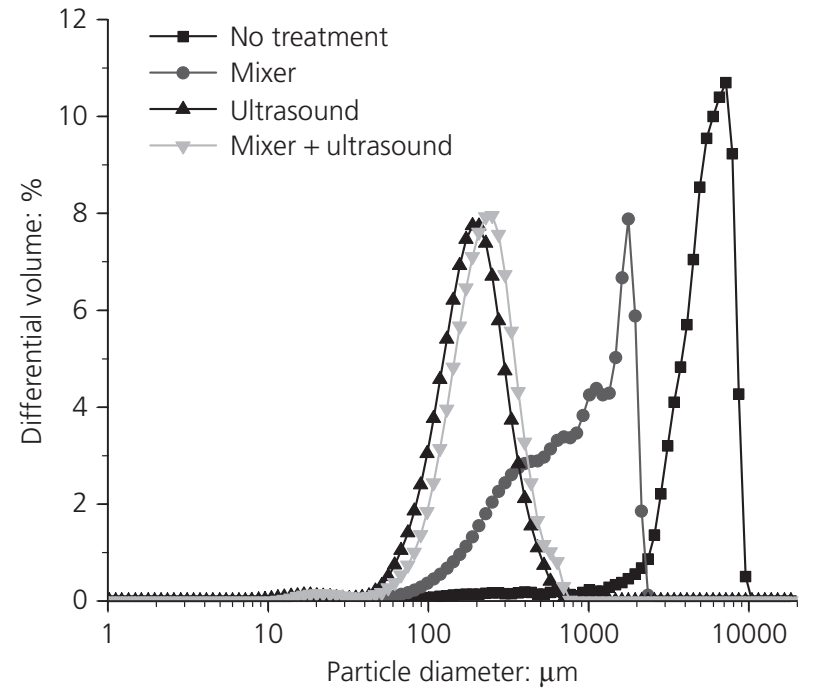

Figure 5. Light scattering results obtained for applied physical treatments

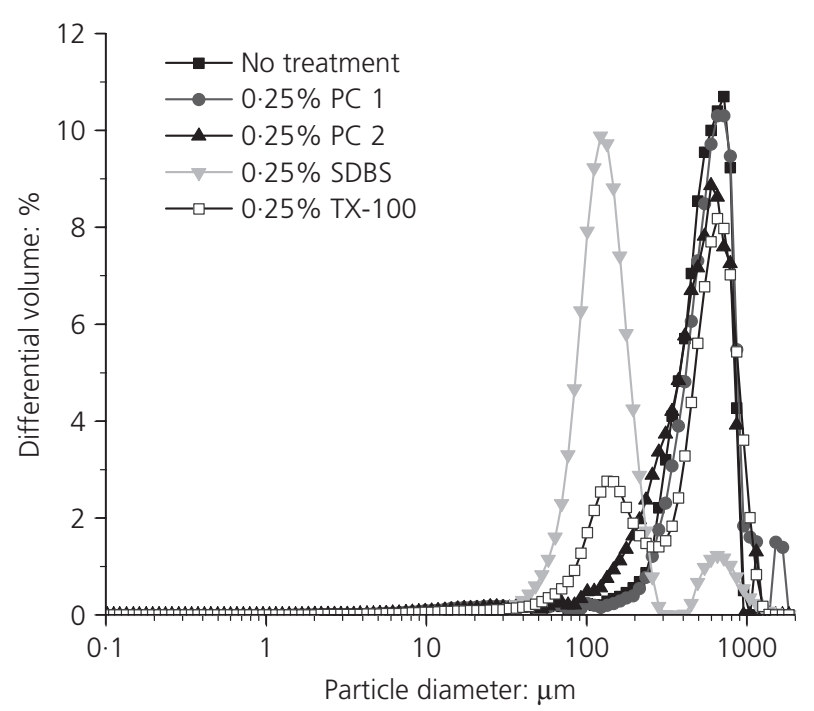

Figure 6. Light scattering results obtained for applied chemical treatments

applied, differences among them are nullified as a result of the synergistic dispersant effect, as shown in Figure 7 for PC1. Results obtained for the combined treatments with the three other dispersants (PC2, SDBS and TX-100) present an identical behaviour as represented for PC1.

The interesting observation obtained from Figure 7 is that, when combining both chemical and ultrasonication techniques, the particle diameter measured decreases considerably, setting up two well-delineated families. One of them has a mean peak of about $2 \mu \mathrm{m}$, which would correspond to a very small bunch of CNTs, the other one is located below $1 \mu \mathrm{m}$, which could correspond to signals of a single CNT. Therefore, the

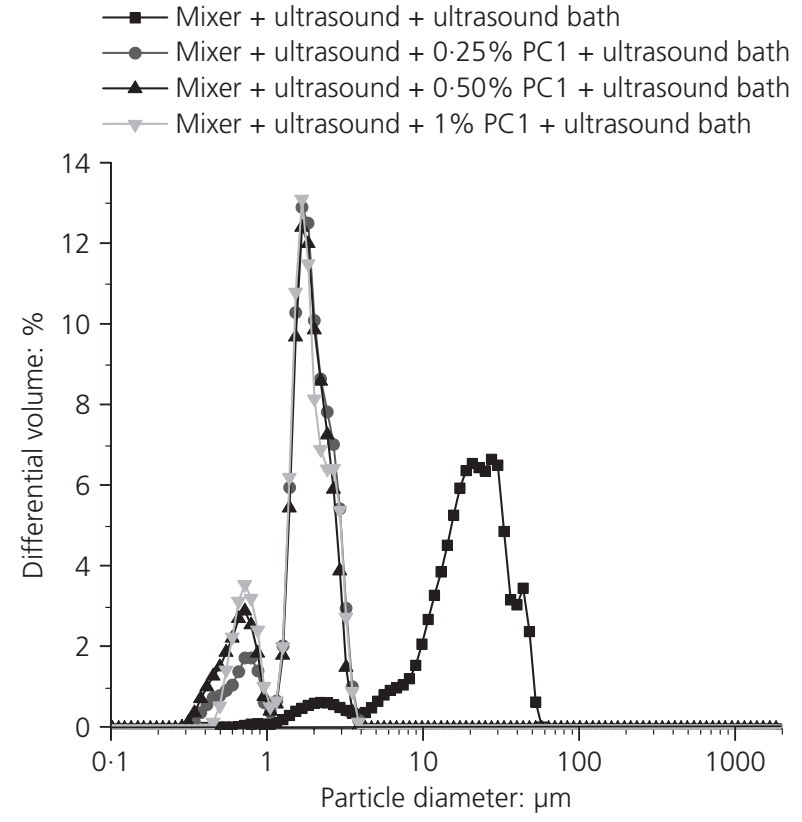

Figure 7. Light scattering results obtained for a combined treatment (physics + different PC1 dosages)

synergistic effect from physical/chemical combination techniques is found to be the best dispersing method for aqueous CNT suspensions. On the other hand, indiscernible differences could be distinguished between four dispersants, and even more, between different dispersant dosages for the fixed CNT amount $(0.05 \%$ by cement mass).

Visual observation confirmed the good stability of combined treatment dispersions, not finding any decantation processes at 7, 30 and even $200 \mathrm{~d}$. The 'mixer' treatment showed a partial decantation at $1 \mathrm{~d}$, meanwhile the 'ultrasound' and 'mixer + ultrasound' physical treatments kept a complete stability also at $200 \mathrm{~d}$. Chemical treatments alone, both surfactants and polycarboxylates, presented an immediate decantation process.

\section{Influence of curing age and current intensity on strain-sensing function}

The strain-sensing tests were performed on the $40 \times 40 \times$ $160 \mathrm{~mm}^{3}$ specimens. To investigate the piezoresistive behaviour of CNT composites the change in electrical resistance was measured under the simultaneous application of a compressive load. Each regular test consisted of at least four consecutive loading and unloading cycles. The values of load, voltage and unit strain were recorded every $1 \mathrm{~s}$, with the experimental procedure lasting from 120 to $720 \mathrm{~s}$ according to the maximum loading and loading rate.

Figure 8 shows the results of strain-sensing tests for the CNT dosages studied $(0 \cdot 05 \%, 0 \cdot 10 \%, 0 \cdot 25 \%$ and $0.50 \% \mathrm{CNT})$, at ages of 14 and $28 \mathrm{~d}$. The maximum stress reached during these tests was $3.75 \mathrm{MPa}$ and the loading rate was $400 \mathrm{~N} / \mathrm{s}$. 


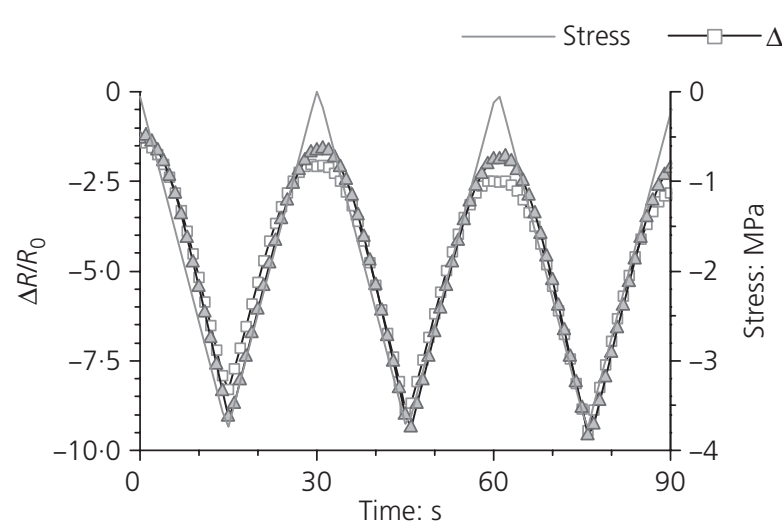

(a)

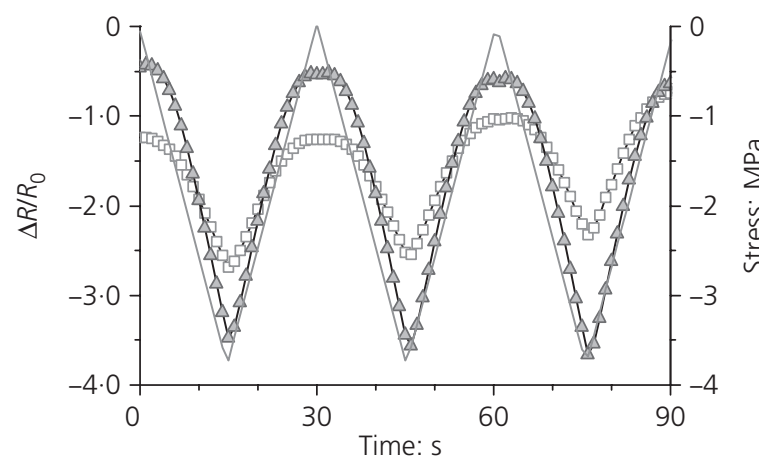

(c)

Figure 8. Strain-sensing tests for different CNT dosages $0.05 \%$, $0.10 \%, 0.25 \%$ and $0.50 \%$ CNT at curing ages of 14 and $28 \mathrm{~d}$. Fractional change on electrical resistance, $\Delta R / R_{0}$ and compressive

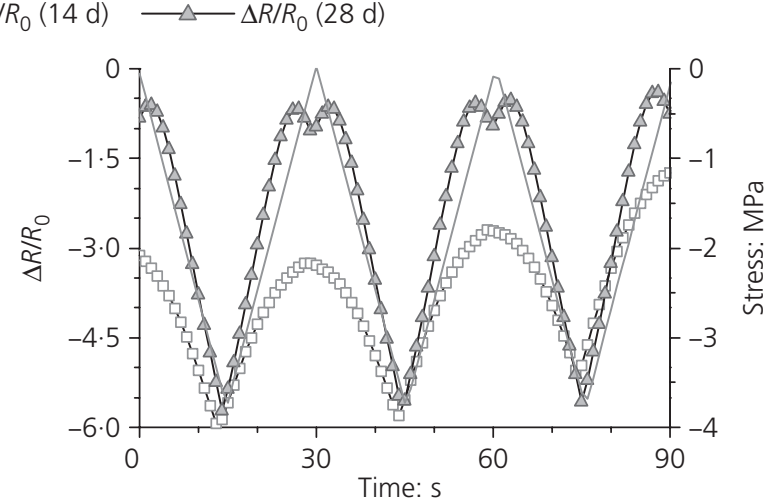

(b)

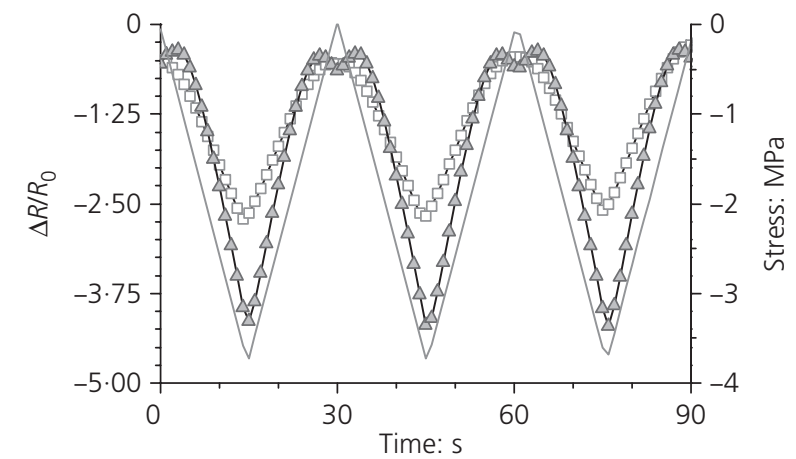

(d)

stress are both represented against time. All tests were run at a constant loading rate of $400 \mathrm{~N} / \mathrm{s}$ and a current intensity of $1 \mathrm{~mA}$ : (a) $0.05 \%$ CNT; (b) $0.10 \%$ CNT; (c) 0.25 CNT; (d) $0.5 \%$ CNT

Fractional changes in electrical resistance and compressive stress are both represented against time. A relationship between the stress and electrical response could be detected in any of the tests from the earliest tested age of $14 \mathrm{~d}$, for whichever current intensity was applied. However, the strain-sensing performance shows a more sensitive behaviour at the age of $28 \mathrm{~d}$ for all dosages, since the reversibility on the measures and the correlation between resistance variation and compressive strain are much better at $28 \mathrm{~d}$ than at $14 \mathrm{~d}$, with the exception of the control sample whose resistance changes are not able to be considered reversible. As it has been previously reported (Chen et al., 2014), cement composites with no conductive admixture do not show self-sensing behaviour, or this phenomenon is neither reversible nor repeatable. However, from the lowest CNT addition to the highest, a fractional change in electric resistance has been obtained, which is well correlated with the stress applied to the specimen. This fractional change in resistance shows distinct magnitude for the different CNT dosages, which in turn means different GF for different CNT dosages.

Figure 9 shows the results of strain-sensing tests for $0.50 \% \mathrm{CNT}$ dosage at a curing age of $28 \mathrm{~d}$. Current intensity was established at three different ranges: $0 \cdot 1 \mathrm{~mA}, 1 \mathrm{~mA}$ and $10 \mathrm{~mA}$. The maximum stress reached during these tests was 5.625 MPa and

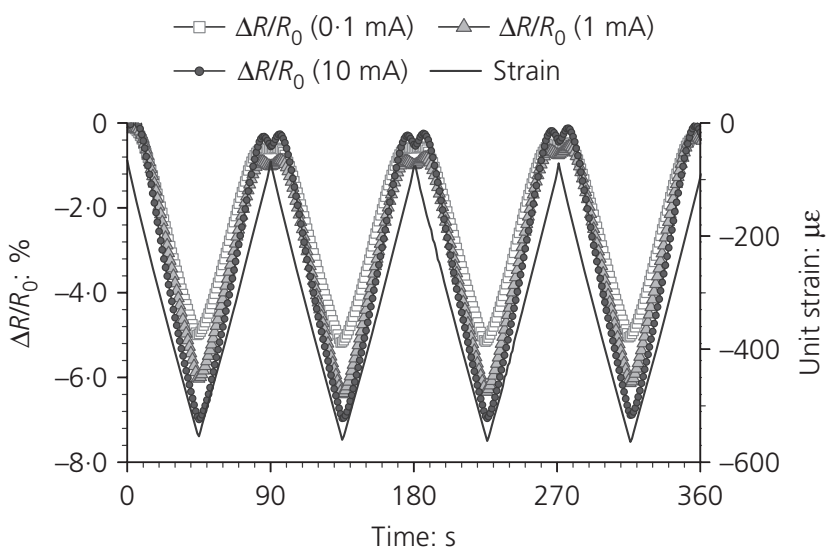

Figure 9. Fractional change in electrical resistance $\left(\Delta R / R_{0}\right)$ and longitudinal unit strain $(\mu \varepsilon)$ plotted against time for $0.50 \%$ CNT cement pastes strain-sensing tests, at $28 \mathrm{~d}$, for $0.1 \mathrm{~mA}, 1.0 \mathrm{~mA}$ and $10.0 \mathrm{~mA}$ current intensities, at a maximum load of $9 \mathrm{kN}$ (5.625 MPa) and a loading rate of $200 \mathrm{~N} / \mathrm{s}$

the loading rate was $200 \mathrm{~N} / \mathrm{s}$. The fractional changes of electrical resistance and compressive stress are both plotted against time. Strain-sensing behaviour is clearly noticeable for all three 
intensities. It has been observed for all CNT additions that all different currents make it possible to obtain a good reversibility on the measures and establish the correlation between resistance variation and compressive strain, and hence to calculate the corresponding GF. However, sensitivity appears to be enhanced when the current intensity increases. This indicates that, apart from requiring a certain current density for the piezoresistive behaviour to be visible (Galao et al., 2014), there is an optimal current density which enables the best correlation levels between electric and mechanical parameters to be obtained.

\section{Effect of loading conditions on strain-sensing function}

In the elastic range pastes behave according to $\sigma=E \varepsilon$, where $\sigma$ is stress (MPa), $E$ is elastic modulus $(\mathrm{MPa})$ and $\varepsilon$ is unit strain. For a specific stress, the registered unit strain will depend on the elastic modulus. So given the influence of $\varepsilon$ for GF calculation, it is also convenient to take elastic modulus values into account. Table 3 shows the mean elastic modulus $(E)$ obtained at the age of $28 \mathrm{~d}$ for a maximum load of $9 \mathrm{kN}$ and loading rate of $100 \mathrm{~N} / \mathrm{s}$. Slight differences were observed, showing the highest $E$ value for the control sample and the lowest elastic modulus for the lowest CNT dosage. These slight differences could be explained by different plasticiser quantities added to each CNT dosage that enable the same workability but also can generate a small increase of matrix stiffness. However, given the similarity of $E$ values for pastes incorporating CNT, a similar mechanical behaviour can be expected for these samples when they are subjected to stress during strain-sensing tests. Regarding the strain distribution in specimens, all samples are expected to experience the same uniaxial tensile stress since they are geometrically equal. Research is presently underway within a structural reinforced element, which also includes other sorts of sensors that are different in shape.

The loading conditions refer to the combination of different maximum loadings $(3,6,9 \mathrm{kN})$ and loading rates $(50,100$, 200 and $400 \mathrm{~N} / \mathrm{s}$ ). After carrying out the nine combinations established in the experimental programme, the pattern plotted in Figure 10 was seen repeatedly. It shows the gauge factor of

\begin{tabular}{lcrc}
\hline $\begin{array}{l}\% \text { CNT } \\
\text { (by cement mass) }\end{array}$ & $\begin{array}{c}\text { Elastic modulus: } \\
\mathrm{N} / \mathrm{mm}^{2}\end{array}$ & $\begin{array}{c}\mathrm{SD}: \\
\mathrm{N} / \mathrm{mm}^{2}\end{array}$ & $\begin{array}{c}\text { RSD: } \\
\%\end{array}$ \\
\hline 0.00 & 12422 & 263 & $2 \cdot 1$ \\
0.05 & 13101 & 235 & $1 \cdot 8$ \\
0.10 & 13574 & 52 & 0.4 \\
0.25 & 10989 & 396 & 3.6 \\
0.50 & 10705 & 318 & 3.0
\end{tabular}

Table 3. Mean elastic modulus $(E)$ obtained for fixed load conditions: maximum load of $9 \mathrm{kN}$ and loading rate of $100 \mathrm{~N} / \mathrm{s}$. Average value, standard deviation (SD) and relative SD obtained for three measures performed at $28 \mathrm{~d}$

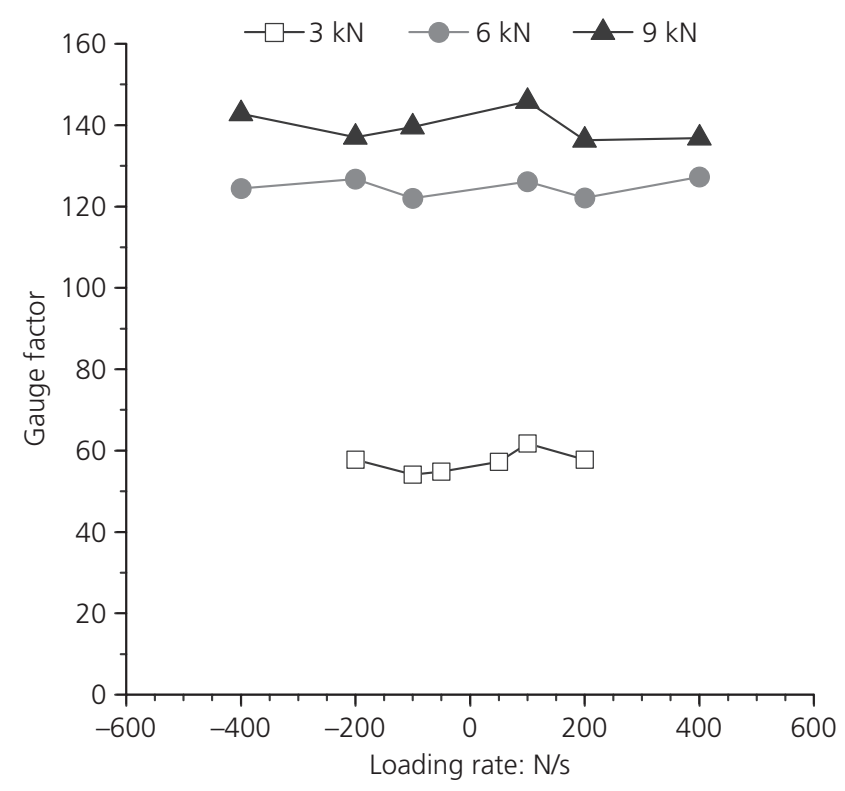

Figure 10. Gauge factor of $0.50 \%$ CNT paste at $28 \mathrm{~d}$ against loading rate (negative values correspond to unloading cycles) for three different maximum axial loads (the stress levels applied were $1.875,3.75$ and $5.625 \mathrm{MPa}$, for 3,6 and $9 \mathrm{kN}$, respectively)

the $0 \cdot 50 \%$ CNT sample for each maximum cycle loading, considering unloading cycles as negative loading rates and loading ones as positive rates. If a certain load level is reached the material's response is independent of the loading rate used (similar conclusions could be drawn for different moving loads' velocities reported in previous research (Baeza et al., 2013a)). This topic is especially important if traffic monitoring applications are desired, because if the materials' response is independent of the loading rate, it will be independent of the vehicle's passing velocity. However, the stress applied to the samples does affect the sensitivity of the CNT cement-based pastes. At the lowest loading level $(3 \mathrm{kN})$ a loss in sensitivity has been registered for all different pastes; that is, composites must experience a minimum strain in order to show improved strain-sensing properties. Besides, once this sensitivity threshold is surpassed the gauge factors continue depending on the stress, but obtain more similar values between the two highest values (6 and $9 \mathrm{kN})$.

Figure 11 includes the mean GF values calculated for specimens with CNT additions, for the maximum load applied of $9 \mathrm{kN}$, that is the maximum load that had been found to show the enhanced sensitivity. Results are shown for the loading rate of $400 \mathrm{~N} / \mathrm{s}$, but for all three different loading rates the obtained behaviour pattern is analogous, according to the non-influence of the loading rate mentioned above. The $R^{2}$ Pearson's coefficient is also plotted, obtained as the average value for both loading and unloading GF linear regressions. Three interesting conclusions can be drawn from these results. 
(a) There were no major differences between loading and unloading cycles, thus the analysis of the mean value of all cycles would be accurate enough

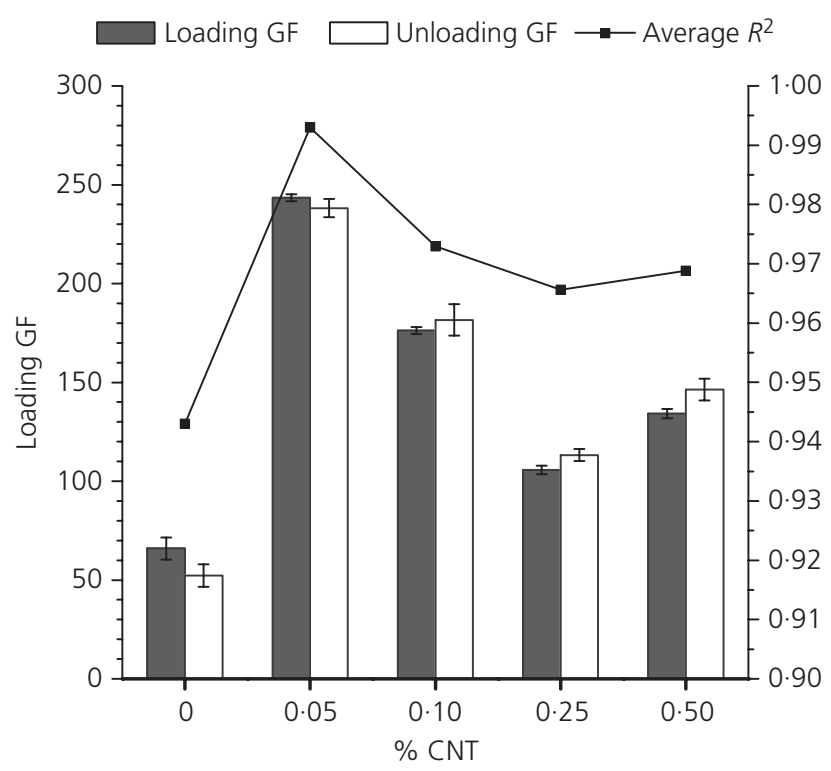

Figure 11. Gauge factor with standard deviation and its $R^{2}$ Pearson's coefficient for different CNT dosages at the age of $28 \mathrm{~d}$. Maximum loading $9 \mathrm{kN}$ and loading rate $400 \mathrm{~N} / \mathrm{s}$. Current intensity established at $1 \mathrm{~mA}$ (b) $R^{2}$ Pearson's coefficient shows a pattern similar to GF values, that is, the higher GF, the better the $R^{2}$ coefficient.

(c) The addition of CNT to the cement paste achieves a gauge factor up to 240, which is reached for the lowest CNT dosage. The most likely explanation for this observation is a better dispersion for $0.05 \% \mathrm{CNT}$ into the matrix. That would mean the same dispersion method applied for all samples is not equally effective for different CNT dosages.

The curves in Figure 12 represent the electrical data (fractional change of resistance) against the mechanical data (strain) when the measures of the same single test (maximum loading $9 \mathrm{kN}$ and loading rate $400 \mathrm{~N} / \mathrm{s}$ ) are represented for different CNT dosages. Linear regressions for the test appear defined for each dosage by their equation, where the slope is GF according to Equation 1, and their $R^{2}$ Pearson's coefficient. Here, the behaviour pattern described for Figure 11 is also identifiable; that is, the highest GF is equally shown by the lowest CNT dosage and it also presents the highest $R^{2}$. At this point the suitability of the GF as a characteristic parameter could be assessed. When analysing the sensing phenomena at the lowest strains, there seems to be a low sensitivity area below $200 \mu \varepsilon$, which would explain the lower GF values for very low stresses. However, when the $200 \mu \varepsilon$ strain value was exceeded, a perfectly linear resistivity change-strain relationship was found. Therefore, according to Equation 1, the GF description would be only available for this second case.

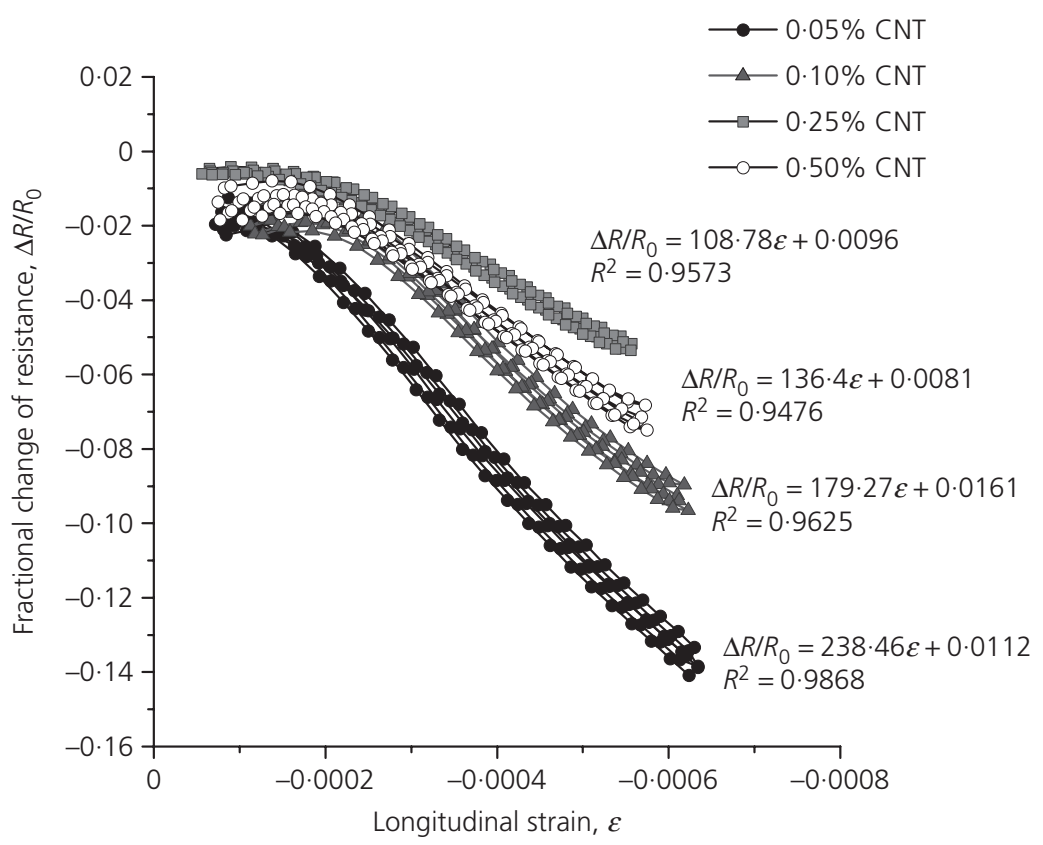

Figure 12. Resistance fractional change $\left(\Delta R / R_{0}\right)$ against longitudinal unit strain $(\varepsilon)$, for different CNT additions at $28 \mathrm{~d}$. intensity established at $1 \mathrm{~mA}$. Linear regression for each CNT Maximum loading $9 \mathrm{kN}$ and loading rate $400 \mathrm{~N} / \mathrm{s}$. Current dosage is included 
In any case, the non-linear singularity at low strains had a small effect on all topics discussed above, as the linear regression curve calculated for every test had high average $R^{2}$ coefficients. Thus GF can actually be used for strain-sensing purposes as proposed by several authors (Baeza et al., 2011; Chung, 2002) (even if third-degree polynomial or sigmoid functions could make better regressions), but certain sensitivity issues should be considered for real applications.

Lastly, as far as durability is concerned, as in previous research (Camacho-Ballesta et al., 2014) it seems to be appropriate to do research into the effect of aggressive conditions, such as carbonation and contamination by chloride ions, in the strainsensing function. New experimental tests are already planned to that effect.

\section{Conclusions}

An insight into the problem of dispersion of CNT has been provided by means of light-scattering particle size analysis. This technique made it possible to discern which treatments presented the best dispersant ability, that is, the combined physical/ chemical treatment (ultrasound + dispersant), but did not show any difference among the distinct dispersants evaluated.

The piezoresistive sensitivity of cement-based CNT sensors was investigated at the ages of 14 and $28 \mathrm{~d}$. Strain-sensing properties were seen in all tests from the earliest tested age of $14 \mathrm{~d}$, whichever current intensity was applied. However, the strainsensing performance showed a more sensitive behaviour at the age of $28 \mathrm{~d}$ for all dosages.

As far as loading conditions were concerned, loading rate did not affect the strain-sensing response of the composites, although the sensitivity (gauge factor) was increased with the maximum compressive load applied.

The best performance as strain sensor was obtained for the $0.05 \%$ CNT composite, reaching values of gauge factor up to 240 with $R^{2}$ Pearson's coefficient above 0.99.

\section{Acknowledgements}

The authors would like to acknowledge the Spanish Ministry of Science and Innovation (Ref: Mat 2009-10866) and Generalitat Valenciana (PROMETEO/2013/035) for their financial support of this research. The authors would also like to thank Bayer MaterialScience, S.A. for the supply of CNTs used in this investigation.

\section{REFERENCES}

AENOR (Spanish Association for Standardisation and Certification) (2005) UNE-EN 196-1:2005. Methods of testing cement - Part 1: Determination of strength. Spanish Association for Standardisation and Certification, Madrid, Spain.
AENOR (2009) UNE-EN 196-3:2005+A1:2009. Methods of testing cement - Part 3: Determination of setting times and soundness. Spanish Association for Standardisation and Certification, Madrid, Spain.

AENOR (2014) UNE-EN 12390-13:2014. Testing hardened concrete - Part 13: Determination of secant modulus of elasticity in compression. Spanish Association for Standardisation and Certification, Madrid, Spain.

Azhari F and Banthia N (2012) Cement-based sensors with carbon fibers and carbon nanotubes for piezoresistive sensing. Cement and Concrete Composites 34(7): 866-873.

Baeza FJ, Galao O, Zornoza E and Garces P (2013a) Effect of aspect ratio on strain sensing capacity of carbon fiber reinforced cement composites. Materials and Design 51(October): 1085-1094.

Baeza FJ, Galao O, Zornoza E and Garces P (2013b) Multifunctional cement composites strain and damage sensors applied on reinforced concrete (RC) structural elements. Materials 6(3): 841-855.

Baeza FJ, Zornoza E, Andion LG, Ivorra S and Garces P (2011) Variables affecting strain sensing function in cementitious composites with carbon fibers. Computers and Concrete 8(2): 229-241.

Bortz DR, Merino C and Martin-Gullon I (2011) Carbon nanofibers enhance the fracture toughness and fatigue performance of a structural epoxy system. Composites Science and Technology 71(1): 31-38.

Camacho-Ballesta MC, Galao O, Baeza FJ, Zornoza E and Garces P (2014) Mechanical properties and durability of CNT cement composites. Materials 7(3): 1640-1651.

Catala G, Ramos-Fernandez EV, Zornoza E, Andion LG and Garces P (2011) Influence of the oxidation process of carbon material on the mechanical properties of cement mortars. Journal of Materials in Civil Engineering 23(3): 321-329.

Chen SJ, Collins FG, Macleod AJN et al. (2011) Carbon nanotube-cement composites: a retrospect. The IES Journal Part A: Civil and Structural Engineering 4(4): 254-265.

Chen SJ, Zou B, Collins F et al. (2014) Predicting the influence of ultrasonication energy on the reinforcing efficiency of carbon nanotubes. Carbon 77(2014): 1-10.

Chung DDL (2002) Piezoresistive cement-based materials for strain sensing. Journal of Intelligent Material Systems and Structures 13(9): 599-609.

Chung DDL (2012) Carbon materials for structural self-sensing, electromagnetic shielding and thermal interfacing. Carbon 50(9): 3342-3353.

Coleman JN, Khan U, Blau WJ, Gun'Ko YK (2006) Small but strong: a review of the mechanical properties of carbon nanotube-polymer composites. Carbon 44(9): 1624-1652.

Collins F, Lambert J and Duan WH (2012) The influences of admixtures on the dispersion, workability, and strength of 
carbon nanotube-OPC paste mixtures. Cement and Concrete Composites 34(2): 201-207.

Galao O, Zornoza E, Baeza FJ, Bernabeu A and Garces P (2012) Effect of carbon nanofiber addition in the mechanical properties and durability of cementitious materials. Materiales de Construccion 62(307): 343-357.

Galao O, Baeza FJ, Zornoza E and Garces P (2014) Strain and damage sensing properties on multifunctional cement composites with CNF admixture. Cement and Concrete Composites 46(2014): 90-98.

Han B, Yu X and Kwon E (2009) A self-sensing carbon nanotube/cement composite for traffic monitoring. Nanotechnology 20(44): 445501.

Han B, Yu X, Kwon E and Ou J (2012) Effects of CNT concentration level and water/cement ratio on the piezoresistivity of CNT/cement composites. Journal of Composite Materials 46(1): 19-25.

Kim HK, Nam IW and Lee HK (2014) Enhanced effect of carbon nanotube on mechanical and electrical properties of cement composites by incorporation of silica fume. Composite Structures 107(January): 60-69.

Konsta-Gdoutos MS and Aza CA (2014) Self sensing carbon nanotube $(\mathrm{CNT})$ and nanofiber $(\mathrm{CNF})$ cementitious composites for real time damage assessment in smart structures. Cement and Concrete Composites 53(October): 162-169.

Li GY, Wang PM and Zhao X (2007) Pressure-sensitive properties and microstructure of carbon nanotube reinforced cement composites. Cement and Concrete Composites 29(5): 377-382.

Luo J, Duan Z and Li H (2009) The influence of surfactants on the processing of multi-walled carbon nanotubes in reinforced cement matrix composites. Physica Status Solidi A 206(12): 2783-2790.

Luo J, Duan Z, Zhao T and Li Q (2011) Effect of compressive strain on electrical resistivity of carbon nanotube cement-based composites. Key Engineering Materials 483 : 579-583.

Parveen S, Rana S and Fangueiro R (2013) A review on nanomaterial dispersion, microstructure, and mechanical properties of carbon nanotube and nanofiber reinforced cementitious composites. Journal of Nanomaterials 2013(2013): 710175.

Wen S and Chung DDL (2006) Model of piezoresistivity in carbon fiber cement. Cement and Concrete Research 36(10): 1879-1885.

Yu X and Kwon E (2009) A carbon nanotube/cement composite with piezoresistive properties. Smart Materials and Structures 18(5): 055010

\section{WHAT DO YOU THINK?}

To discuss this paper, please submit up to 500 words to the editor at journals@ice.org.uk. Your contribution will be forwarded to the author(s) for a reply and, if considered appropriate by the editorial panel, will be published as a discussion in a future issue of the journal. 